\title{
miR-449a-5p suppresses CDK6 expression to inhibit cardiomyocyte proliferation
}

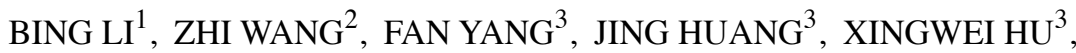 \\ SHIYAN DENG ${ }^{3}$, MAOBO TIAN $^{3}$ and XIAOYUN SI ${ }^{4}$ \\ ${ }^{1}$ School of Medicine, Guizhou University, Guiyang, Guizhou 550025; ${ }^{2}$ Department of Emergency Medicine, \\ Qingdao Municipal Hospital (Group), Qingdao, Shandong 266011; ${ }^{3}$ Department of Cardiology, \\ Guizhou Provincial People's Hospital, Guiyang, Guizhou 550002; ${ }^{4}$ Department of Cardiology, \\ The Affiliated Hospital of Guizhou Medical University, Guiyang, Guizhou 550004, P.R. China
}

Received May 8, 2020; Accepted September 2, 2020

DOI: $10.3892 / \mathrm{mmr} .2020 .11652$

\begin{abstract}
Induction of cardiomyocyte (CM) proliferation is a promising approach for cardiac regeneration following myocardial injury. MicroRNAs (miRs) have been reported to regulate $\mathrm{CM}$ proliferation. In particular, miR-449a-5p has been identified to be associated with $\mathrm{CM}$ proliferation in previous high throughput functional screening data. However, whether miR-449a-5p regulates CM proliferation has not been thoroughly investigated. This study aimed to explore whether miR-449a-5p modulates CM proliferation and to identify the molecular mechanism via which miR-449a-5p regulates CM proliferation. The current study demonstrated that miR-449a-5p expression levels were significantly increased during heart development. Furthermore, the results suggested that miR-449a-5p mimic inhibited CM proliferation in vitro as determined via immunofluorescence for ki67 and histone $\mathrm{H} 3$ phosphorylated at serine $10(\mathrm{pH} 3)$, as well as the numbers of CMs. However, miR-449a-5p knockdown promoted CM proliferation. CDK6 was identified as a direct target gene of miR-449a-5p, and CDK6 mRNA and protein expression was suppressed by miR-449a-5p. Moreover, CDK6 gain-of-function increased CM proliferation. Overexpression of CDK6 also blocked the inhibitory effect of miR-449a-5p on $\mathrm{CM}$ proliferation, indicating that CDK6 was a functional target of miR-449a-5p in CM proliferation. In conclusion, miR-449a-5p inhibited CM proliferation by targeting CDK6, which provides a potential molecular target for preventing myocardial injury.
\end{abstract}

Correspondence to: Dr Xiaoyun Si, Department of Cardiology, The Affiliated Hospital of Guizhou Medical University, 28 Guiyi Road, Guiyang, Guizhou 550004, P.R. China

E-mail: sixiaoyun1@qq.com

Key words: cardiomyocyte proliferation, microRNA-449a-5p, CDK6

\section{Introduction}

Cardiac disease is the most prevalent cause of mortality worldwide (1). Damage to the mammalian heart leads to cardiomyocyte (CM) loss, cardiac fibrosis and hypertrophy followed by cardiac dysfunction, which eventually leads to heart failure (2). Adult mammalian CMs become fully differentiated, exit the cell cycle and are unable to regenerate $\mathrm{CMs}$ following cardiac diseases (3). Therefore, the induction of $\mathrm{CM}$ proliferation presents a promising strategy to promote cardiac regeneration $(4,5)$.

MicroRNAs (miRNAs/miRs) are a group of $\sim 22$-nucleotide, highly conserved, non-coding RNAs that regulate gene expression via post-transcriptional modification in numerous biological and pathological processes, such as cell proliferation and apoptosis (6). Previous studies have revealed that miRNA is a critical regulator in cardiac conditions, including in cardiac hypertrophy, myocardial infarction and heart failure $(7,8)$. To date, multiple miRNAs have been identified that control fetal CM proliferation and postnatal CM cell cycle reentry $(6,9,10)$. A high-throughput functional screening identified that $\sim 204$ miRNAs promoted neonatal CM proliferation, whereas 331 miRNAs decreased CM proliferation (11). The miRNA clusters miR-17-92 (12) and miR-302-367 (9) are reported to be involved in CM proliferation in mouse embryonic and postnatal hearts. Moreover, miR-590-3p and miR-199a-3p increase cyclin A (CCNA), cyclin E (CCNE) and cyclin B (CCNB) expression levels in proliferating CMs (11). It has also been shown that miR-204-dependent regulation of Jumonji And AT-Rich Interaction Domain Containing 2 (Jarid2) in CM is essential during embryo development and cardiogenesis (13).

miR-449a-5p has been reported to be a tumor suppressor in numerous types of cancer including gastric cancer, liver cancer and neuroblastoma (14-16). Previous studies have demonstrated that miR-449a-5p inhibits cell cycle progression, proliferation, growth and invasion in cancer cells by targeting different target genes (17). A previous study also revealed that miR-449a-5p regulated hypoxia/reoxygenation injury-induced CM mortality (18). Furthermore, the members of the miR-34/449 family share high sequence homology (19). miR-34a has been shown to inhibit CM proliferation in 
postnatal mice, whereas inhibition of miR-34a promotes CM proliferation and improves cardiac function in adult post-myocardial infarcted hearts (10). However, to the best of our knowledge there is no direct evidence of miR-449a-5p modulation of CM proliferation.

Integrated bioinformatics analyses have revealed that CDK6 is a potential target of miR-449a-5p (16,20). CDK6 is a cyclin $\mathrm{D}$ activated kinase that phosphorylates the retinoblastoma protein $(\mathrm{Rb})$ in the $\mathrm{G}_{1}$ phase, and associates with $\mathrm{E} 2$ transcription factor $(\mathrm{E} 2 \mathrm{~F})$ to regulate the transition from $G_{1}$ to $S$ in the cell cycle (21). Additionally, previous studies have reported that miR-449a-5p directly targets CDK6 to inhibit cell proliferation $(16,20)$.

Based on the relationship between miR-449a-5p and CDK6, it was hypothesized that miR-449a-5p may suppress CM proliferation by inhibiting CDK6. The aims of the current study were to elucidate whether loss of miR-449a-5p promotes CM proliferation and to identify the molecular mechanism via which miR-449a-5p regulates $\mathrm{CM}$ proliferation. The present study provides a novel insight into miR-449a-5p, which could potentially be used as an effective therapeutic approach in cardiac diseases.

\section{Materials and methods}

Reagents and antibodies. Trypsin, collagenase type II, FBS and Opti-MEM were purchased from Gibco, Thermo Fisher Scientific, Inc. DMEM/F12 1:1 medium was obtained from HyClone, Cytiva. An E.Z.N.A. Total RNA kit II was purchased from Omega Bio-Tek, Inc. An RNeasy Midi kit was acquired from Qiagen, Inc. DNase I and Lipofectamine ${ }^{\circledR} 2000$ were purchased from Invitrogen, Thermo Fisher Scientific, Inc. A PrimeScript ${ }^{\mathrm{TM}}$ RT Master mix and a SYBRs Premix Ex Taq ${ }^{\mathrm{TM}}$ kit were obtained from Takara Biotechnology Co., Ltd. A miRNA First Strand cDNA Synthesis and miRNAs Quantitation PCR kit were purchased from Sangon Biotech Co., Ltd. Paraformaldehyde (PFA) was acquired from Leagene. DAPI was purchased from Bestbio, while Hoechst 33342 was purchased from Bioworld Technology, Inc. Tris-HCl, RIPA buffer was obtained from Beijing Dingguo Changsheng Biotechnology Co., Ltd. A BCA Protein Quantitative Analysis kit was purchased from Fudebio-tech. BSA, penicillin, streptomycin, $\mathrm{NaCl}$, SDS, formamide, and SSC were purchased from Sigma-Aldrich, Merck KGaA.

For immunofluorescence analysis, antibodies against cardiac troponin T (cTnT; cat. no. ab8295; 1:100), ki67 (cat. no. ab15580; 1:100) and histone H3 phosphorylated at serine 10 (pH3; cat. no. ab47297; 1:100) were obtained from Abcam, and goat anti-mouse IgG/Alexa Fluor 488 (cat.no. bs-0296G-A488; 1:100) and goat anti-rabbit IgG/Alexa Fluor 555 (cat. no. bs-0295G-A555; 1:100) antibodies were purchased from Beijing Biosynthesis Biotechnology Co., Ltd.

For western blotting, antibodies against CDK6 (cat.no.3136; 1:2,000) were purchased from Cell Signaling Technology, Inc., and $\beta$-actin (cat. no. bs-0061R; 1:2,000) antibodies were purchased from Beijing Biosynthesis Biotechnology Co., Ltd. Donkey anti-mouse IgG H\&L (cat. no. ab6820; 1:10,000) and donkey anti-rabbit IgG H\&L (cat. no. ab16284; 1:10,000) antibodies were purchased from Abcam.
Laboratory animals. A total of 30 pregnant, 500 postnatal day (P) 1 (1-2 g), 30 P4 (3-4 g), 80 P7 (5-6 g), 20 P28 (18-20 g) and 20 P56 male (20-25 g) C57BL/6J mice were purchased from the Experimental Animal Center of Guizhou Medical University. Animals were housed in a temperature-controlled $\left(23 \pm 1^{\circ} \mathrm{C}\right)$, humidity-controlled (40-60\%) environment with a 12-h light/dark cycle and food and water available ad libitum. All animal experiments were approved by the Guizhou University Subcommittee of Experimental Animal Ethics (Guizhou, China). The current study followed the guidelines of the Guide for the Care and Use of Laboratory Animals published by the US National Institutes of Health (22).

Tissue collection. Ventricular heart tissues were isolated from embryonic day 16.5 (E16.5), P1, P7, P28 and P56 C57BL/6J mice. Mice were deeply anesthetized with $2 \%$ inhaled isoflurane and euthanized by cervical dislocation. Then, hearts were dissected out and washed with $0.9 \% \mathrm{NaCl}$. The hearts were further processed for total RNA isolation and reverse transcription-quantitative PCR (RT-qPCR).

Ventricular CM and cardiac fibroblast (CF) isolation. CMs were isolated on $\mathrm{P} 1, \mathrm{P} 4$ or $\mathrm{P} 7$ from $\mathrm{C} 57 \mathrm{BL} / 6 \mathrm{~J}$ mice, as previously described $(10,11)$. Briefly, the mice were anesthetized using $2 \%$ isoflurane inhalation. The ventricles were separated from the atria, cut into pieces and digested with $0.25 \%$ trypsin at $4^{\circ} \mathrm{C}$ overnight. Then, digestion was performed two to three times using $10 \mathrm{mg}$ collagenase type II and $50 \mathrm{mg}$ BSA in $10 \mathrm{ml}$ PBS at $37^{\circ} \mathrm{C}$ for $15 \mathrm{~min}$ under constant stirring. Following digestion, the supernatant was collected with DMEM/F12 1:1 medium supplemented with $10 \%$ FBS. The collected supernatant was centrifuged $(500 \mathrm{x} \mathrm{g})$ at room temperature for $5 \mathrm{~min}$ to harvest the cells, which were resuspended in DMEM/F12 1:1 medium supplemented with $10 \%$ FBS, $100 \mathrm{U} / \mathrm{ml}$ penicillin and $100 \mathrm{mg} / \mathrm{ml}$ streptomycin. The collected cells, containing $\mathrm{CMs}$ and CFs were seeded onto 100-mm plastic dishes for $2 \mathrm{~h}$ at $37^{\circ} \mathrm{C}$ in a humidified atmosphere of $5 \% \mathrm{CO}_{2}$. CMs and $\mathrm{CFs}$ were cultured via differential adhesion, as described previously $(23,24)$. The CFs adhered to the uncoated cell-culture dish. The supernatant, composed mostly of CMs, was then collected and pelleted. Following this, the CMs were resuspended in DMEM/F12 containing 10\% FBS, counted using a BA210 Digital light microscope (magnification, x100), and plated at the appropriate density (70-80\% confluency).

$R N A$ isolation and RT-qPCR. Total RNA was isolated from cultured P1, P4, or P7 CMs or E16.5, P1, P7, P28 or P56 ventricular heart tissue using an E.Z.N.A. Total RNA kit II, according to the manufacturer's protocol. Nuclear and cytoplasmic RNAs were isolated using an RNeasy Midi kit according to the manufacturer's protocol. RNAs were treated with DNase I ( $1 \mu \mathrm{l}$ for $1 \mu \mathrm{g}$ RNA) for $15 \mathrm{~min}$ at room temperature to exclude DNA contamination.

For the quantification of mRNA expression, PrimeScript ${ }^{\mathrm{TM}}$ RT Master mix was used, according to the manufacturer's protocol, to synthesize cDNA at $37^{\circ} \mathrm{C}$ for $15 \mathrm{~min}$ and $85^{\circ} \mathrm{C}$ for $5 \mathrm{sec}$. RT-qPCR was then performed with a SYBRs Premix Ex Taq ${ }^{\mathrm{TM}}$ kit on a Lightcycler 480 (Roche Diagnostics). A total of $20 \mu 1$ reaction mixture was incubated at $95^{\circ} \mathrm{C}$ for $30 \mathrm{sec}$ for initial denaturation followed by $95^{\circ} \mathrm{C}$ for $5 \mathrm{sec}$ and $60^{\circ} \mathrm{C}$ for $30 \mathrm{sec}$ 
for 40 cycles. To normalize gene expression, $\beta$-actin was used as the reference gene. For the quantification of miRNA expression, miRNA First Strand cDNA Synthesis and miRNAs Quantitation PCR kits were used according to the manufacturers' protocol. U6 was used as a reference gene to normalize miRNA expression, according to previous studies $(25,26)$.

mRNA and miRNA relative expression levels were analyzed using the $2^{-\Delta \Delta \mathrm{Cq}}$ method (27). The difference between the $\mathrm{Cq}$ values of the target gene and internal reference gene in each group were used as the $\Delta \mathrm{Cq}$ of each group, and the average value of $\Delta \mathrm{Cq}$ of each group was subtracted from that of the control group to obtain the $\Delta \Delta \mathrm{Cq}$ of each group; next, $2^{-\Delta \Delta \mathrm{Cq}}$ was used to calculate the relative expression level of each group. To determine the endogenous efficiency, cDNA samples were diluted to four concentration gradients (cDNA dilution: $0.001,0.01,0.1,1)$, amplified with primers specific to $\beta$-actin and CDK6 and the average $\mathrm{Cq}$ values of $\beta$-actin and CDK6, as well as the $\Delta \Delta \mathrm{Cq}$ values, were calculated. Finally, $\Delta \Delta \mathrm{Cq}$ values were mapped using log values of the cDNA concentration gradient. If the absolute slope of the obtained straight line is close to zero, the amplification efficiency of $\beta$-actin and CDK6 primers is equal. All primers were designed by Sangon Biotech Co., Ltd. The primer sequences are provided in Table I.

Fluorescence in situ hybridization (FISH). Isolated CMs were cultured on coverslips, fixed in $4 \%$ PFA for $30 \mathrm{~min}$ and washed with PBS at room temperature. Following this, cells were permeabilized in $0.2 \%$ Triton X-100 in PBS for $10 \mathrm{~min}$ at room temperature, washed with PBS three times, hybridized with a hybridization solution (containing $20 \mathrm{mM}$ Tris- $\mathrm{HCl}, 0.9 \mathrm{M} \mathrm{NaCl}, 0.01 \%$ SDS, and $40 \%$ formamide) and incubated with $10 \mu \mathrm{M}$ labeled miR-449a-5p probe at $37^{\circ} \mathrm{C}$ for $12 \mathrm{~h}$. The miR-449a-5p probe was synthesized by Sangon Biotech Co., Ltd. The cells were washed with 2 X SSC (5 min), $1 \mathrm{X} \mathrm{SSC} \mathrm{(5} \mathrm{min)} \mathrm{and} \mathrm{0.5X} \mathrm{SSC} \mathrm{(5} \mathrm{min)} \mathrm{at} \mathrm{room} \mathrm{temperature,}$ and incubated with DAPI for $2 \mathrm{~h}$ at $37^{\circ} \mathrm{C}$. Image acquisition was performed using an LSM 880 confocal microscope (magnification, x1,000; Zeiss GmbH).

Transfection of plasmids, miRNA mimics and inhibitors. The plasmid pcDNA-CDK6 and pcDNA-NC was synthesized by Vigene. miR-449a-5p mimics, mimic-NCs (cat. no. miR1N0000001-1-5), miR-449a-5p inhibitors, and inhibitor-NCs (cat. no. miR2N0000001-1-5) were synthesized by Guangzhou Ribobio Co.,Ltd. The sequences of miR-449a-5p mimics were 5'-UGGCAGUGUAUUGUUAGCUGGU-3'. The sequences of miR-449a-5p inhibitors were 5'-ACCAGCUAA CAAUACACUGCCA-3'. After isolated P1 CMs were cultured for $48 \mathrm{~h}$ at $37^{\circ} \mathrm{C}, 5 \mu \mathrm{l}$ Lipofectamine ${ }^{\circledR} 2000$ and $50 \mathrm{nM}$ plasmids, mimics or inhibitors were added to Opti-MEM. The mixture was added to the cells following incubation at room temperature for $20 \mathrm{~min}$. This medium was replaced after $6 \mathrm{~h}$ at $37^{\circ} \mathrm{C}$ with the same volume of DMEM/F12 medium. RNA or protein was isolated and immunofluorescence analysis was conducted following $48 \mathrm{~h}$.

Immunofluorescence analysis. For cultured CMs in 24-well plates, the culture medium was washed with PBS, fixed with $4 \%$ PFA for $30 \mathrm{~min}$ at room temperature, permeabilized with
$0.2 \%$ Triton $\mathrm{X}-100$ in PBS for $10 \mathrm{~min}$ at room temperature, and blocked with PBS containing 1\% BSA at room temperature for $30 \mathrm{~min}$. Cells were then incubated with primary antibodies, including cTnT, ki67 or $\mathrm{pH} 3$, for $2 \mathrm{~h}$ at room temperature, followed by washing with PBS and incubation with goat anti-mouse IgG/Alexa Fluor 488 or goat anti-rabbit IgG/Alexa Fluor 555 secondary antibodies for $1 \mathrm{~h}$ at room temperature. Subsequently, cells were washed with PBS and incubated with Hoechst 33342 at room temperature for $30 \mathrm{~min}$. Apoptotic cell death was determined via TUNEL staining (Roche Diagnostics) at room temperature for $1 \mathrm{~h}$, according to the manufacturer's protocol. Image acquisition was performed using an LSM 880 confocal microscope (magnification, x200). To quantify CM proliferation, ki67 and $\mathrm{pH} 3$ marked cell proliferation, while cTnT labeled CM areas. Hoechst 33342 was used for nuclear counterstaining. Quantitative data were obtained by measuring co-localization of Hoechst 33342 staining with ki67 or pH3 in cTnT areas using ImageJ software (version no. 1.6.0; National Institutes of Health). In total, four fields of each section were randomly selected to examine for quantification.

Luciferase reporter assay. Luciferase reporter assays were performed as previously described (28). Wild-type (WT) CDK6 3'-untranslated region (UTR; CDK6-WT) and CDK6 3'-UTR mutant (MUT) derivatives devoid of the miR-449a-5p binding site (CDK6-MU1 and CDK6-MU2) were cloned into the psiCHECKTM-2 vector (SaichengBio Co. Ltd.). After isolated CMs were cultured for $48 \mathrm{~h}, 5 \mu \mathrm{l}$ Lipofectamine ${ }^{\circledR} 2000$ and $50 \mathrm{nM}$ mimic-miR-449a-5p, CDK6-WT or CDK6-MU plasmids were added to Opti-MEM. The mixture was added to the cells following incubation at room temperature for $20 \mathrm{~min}$. The medium was replaced after incubation for $6 \mathrm{~h}$ at $37^{\circ} \mathrm{C}$ with the same volume of DMEM/F12 medium. Cells were harvested at $48 \mathrm{~h}$ post-transfection and luciferase activity was determined using a luciferase reporter system (Promega Corporation), according to the manufacturer's protocol. The firefly luciferase activity was normalized to Renilla luciferase activity

Western blotting. Isolated CMs were lysed in ice-cold RIPA with protease inhibitors and protein concentrations were detected using a BCA Protein Quantitative Analysis kit. A total of $30 \mu \mathrm{g}$ of protein samples were electrophoresed using 10\% SDS-PAGE and transferred to PVDF membranes (EMD Millipore). The PVDF membranes were incubated in blocking buffer [5\% BSA in TBS-0.1\% Tween-20-(TBST) buffer] for $2 \mathrm{~h}$ at room temperature. Following blocking, the PVDF membranes were incubated with primary antibodies (CDK6, $36 \mathrm{kDa}$ or $\beta$-actin, $42 \mathrm{kDa}$ ) in $5 \% \mathrm{BSA}$ at $4^{\circ} \mathrm{C}$ overnight. After washing with TBST, the PVDF membranes were incubated with donkey anti-mouse IgG H\&L or donkey anti-rabbit IgG H\&L antibodies for $1 \mathrm{~h}$ at room temperature. Images were acquired using the chemiluminescence imager GeneGnome XRQ (Syngene). ImageJ software (version no. 1.6.0; National Institutes of Health) was used to calculate the relative density. The intensity of each protein was normalized to $\beta$-actin.

miRNA target prediction. Putative miRNA targets were identified using the miRNA target prediction tools miRmap (version no. 1.1) (29) and miRanda (version no. 3.3) (30). 
Table I. List of the sequences of the primers used in the current study.

\begin{tabular}{lll}
\hline Primer & \multicolumn{1}{c}{ Forward $\left(5^{\prime} \rightarrow 3^{\prime}\right)$} & Reverse $\left(5^{\prime} \rightarrow 3^{\prime}\right)$ \\
\hline miR-449a-5p reverse transcription primer & GTCGTATCCAGTGCAGGGTCCGAGGTATTCGCACTGGATACGACACCAGC \\
miR-449a-5p & CGCGCGTGGCAGTGTATTGTTA & ATCCAGTGCAGGGTCCGAGG \\
U6 & CTCGCTTCGGCAGCACA & AACGCTTCACGAATTTGCGT \\
CDK6 & TAGCTGTCTCCACCACCCAC & GGCCATCTGTCGTTAGCCAG \\
RUNX1 & AACCAGGTAGCGAGATTCAACGAC & CAACTTGTGGCGGATTTGTAAAGA \\
c-kit & CGGGCTAGCCAGAGACATCA & TCTCTGGTGCCATCCACTTCA \\
DAB2 & TAGTCCAACAGAAAGCAAAG & GAGGTGACTCCATTTGTTAAG \\
$\beta$-actin & TGCTGTCCCTGTATGCCTCTG & TTGATGTCACGCACGATTTCC
\end{tabular}

miR, microRNA; RUNX1, Runt-related transcription factor 1; c-kit, mast/stem cell growth factor receptor kit; DAB2, disabled homolog 2.

Statistical analysis. All experiments were repeated at least three times. Data are presented as the mean \pm SEM. Statistical analyses were performed using SPSS software (version no. 20.0; IBM Corp.). For comparison between two groups, an unpaired two-tailed Student's t-test was used. For the comparison of $\geq 3$ groups, one-way ANOVA followed by the Least Significant Difference or Tukey's post hoc test was used. For correlation analysis, Spearman's test was used. $\mathrm{P}<0.05$ was considered to indicate a statistically significant difference.

\section{Results}

miR-449a-5p is upregulated in aged mouse CMs. Firstly, the expression levels of miR-449a-5p in embryonic day 16.5 (E16.5), P1, P7, P28 and P56 were detected ventricular heart tissues. RT-qPCR results demonstrated that miR-449a-5p expression was significantly increased during cardiac development (Fig. 1A). Additionally, miR-449a-5p expression progressively increased in isolated $\mathrm{P} 1, \mathrm{P} 4$ and $\mathrm{P} 7 \mathrm{CMs}$ (Fig. 1B). These results suggested that miR-449a-5p expression gradually increased with age. miR-449a-5p was highly expressed in CMs compared with CFs (Fig. 1C). RT-qPCR results identified that miR-449a-5p was mainly expressed in the cytoplasm of CMs (Fig. 1D). This was further demonstrated via FISH assays results, which indicated that $\mathrm{miR}-449 \mathrm{a}-5 \mathrm{p}$ was primarily located in the cytoplasm of CMs (Fig. 1E).

Overexpression of $m i R-449 a-5 p$ inhibits $C M$ proliferation. To investigate the potential role of miR-449a-5p in CM proliferation, isolated $\mathrm{P} 1 \mathrm{CMs}$ were transfected with mimic-NCs or mimic-miR-449a-5p. RT-qPCR results demonstrated that mimic-miR-449a-5p significantly overexpressed miR-449a-5p in isolated P1 CMs compared with the mimic-NC group (Fig. 2A). Following this, CM proliferation was examined using the cell cycle activity marker ki67 and the mitosis marker pH3. Compared with the mimic-NC, miR-449a-5p overexpression significantly decreased the percentage of ki67-positive CMs $(6.58 \pm 0.38 \%$ vs. $3.47 \pm 0.33 \%$, respectively; Fig. $2 \mathrm{~B})$ and the percentage of $\mathrm{pH} 3$-positive CMs $(1.30 \pm 0.18 \%$ vs. $0.59 \pm 0.24 \%$, respectively; Fig. 2C). Furthermore, miR-449a-5p overexpression decreased the number of $\mathrm{P} 1 \mathrm{CMs}$ to $0.85 \pm 0.04$-fold (Fig. 2D).
Whether miR-449a-5p had an effect on CM dedifferentiation was then examined. Previous studies have reported that the stem/progenitor markers Runt related transcription factor 1 (RUNX1), mast/stem cell growth factor receptor kit (c-kit), and differentially-expressed protein 2 (DAB2) identify dedifferentiated CMs $(31,32)$. RT-qPCR results indicated that miR-449a-5p mimic significantly inhibited RUNX1, c-kit and DAB2 expression levels in isolated P1 CMs compared with the mimic-NC group (Fig. S1A), suggesting that miR-449a-5p inhibited CM dedifferentiation. TUNEL staining results identified that miR-449a-5p overexpression significantly increased isolated P1 CM apoptosis compared with the mimic-NC group (Fig. S1B). These findings demonstrated that miR-449a-5p may be an endogenous regulator of neonatal CM proliferation.

Knockdown of miR-449a-5p stimulates CM proliferation. Having established that miR-449a-5p overexpression inhibited $\mathrm{CM}$ proliferation, it was then evaluated whether knockdown of miR-449a-5p had an effect on CM proliferation. Transfection of isolated P1 CMs with inhibitor-miR-449a-5p led to a significant decrease in miR-449a-5p expression compared with the inhibitor-NC group (Fig. 3A). The effect of miR-449a-5p knockdown on CM proliferation was examined using ki67 and $\mathrm{pH} 3$ immunofluorescence staining. Compared with the inhibitor-NC group, silencing of miR-449a-5p significantly increased the number of ki67-positive $(6.93 \pm 0.41 \%$ vs. $13.55 \pm 0.56 \%$, respectively; Fig. 3B) and $\mathrm{pH} 3$-positive CMs $(1.32 \pm 0.14 \%$ vs. $2.64 \pm 0.20 \%$; Fig. 3 C). Compared with the inhibitor-NC group, miR-449a-5p knockdown demonstrated a 1.23 \pm 0.04 -fold increase in the numbers of CMs (Fig. 3D). These data indicated that $\mathrm{miR}-449 \mathrm{a}-5 \mathrm{p}$ regulated neonatal $\mathrm{CM}$ proliferation.

CDK6 is a direct target gene of miR-449a-5p. Since miRNAs regulate biological function mainly by binding to the 3'-UTR of the target miRNAs (33), miRmap (29) and miRanda (30) were used to perform a bioinformatics search. CDK6, an important cell cycle regulator that induces cell cycle progression and cell proliferation $(34,35)$, was identified as a potential target of miR-449a-5p (Fig. 4A). Using a reporter construct with the putative miR-449a-5p binding site of CDK6 3'-UTR downstream of the luciferase gene, the results demonstrated that miR-449a-5p overexpression significantly decrease 

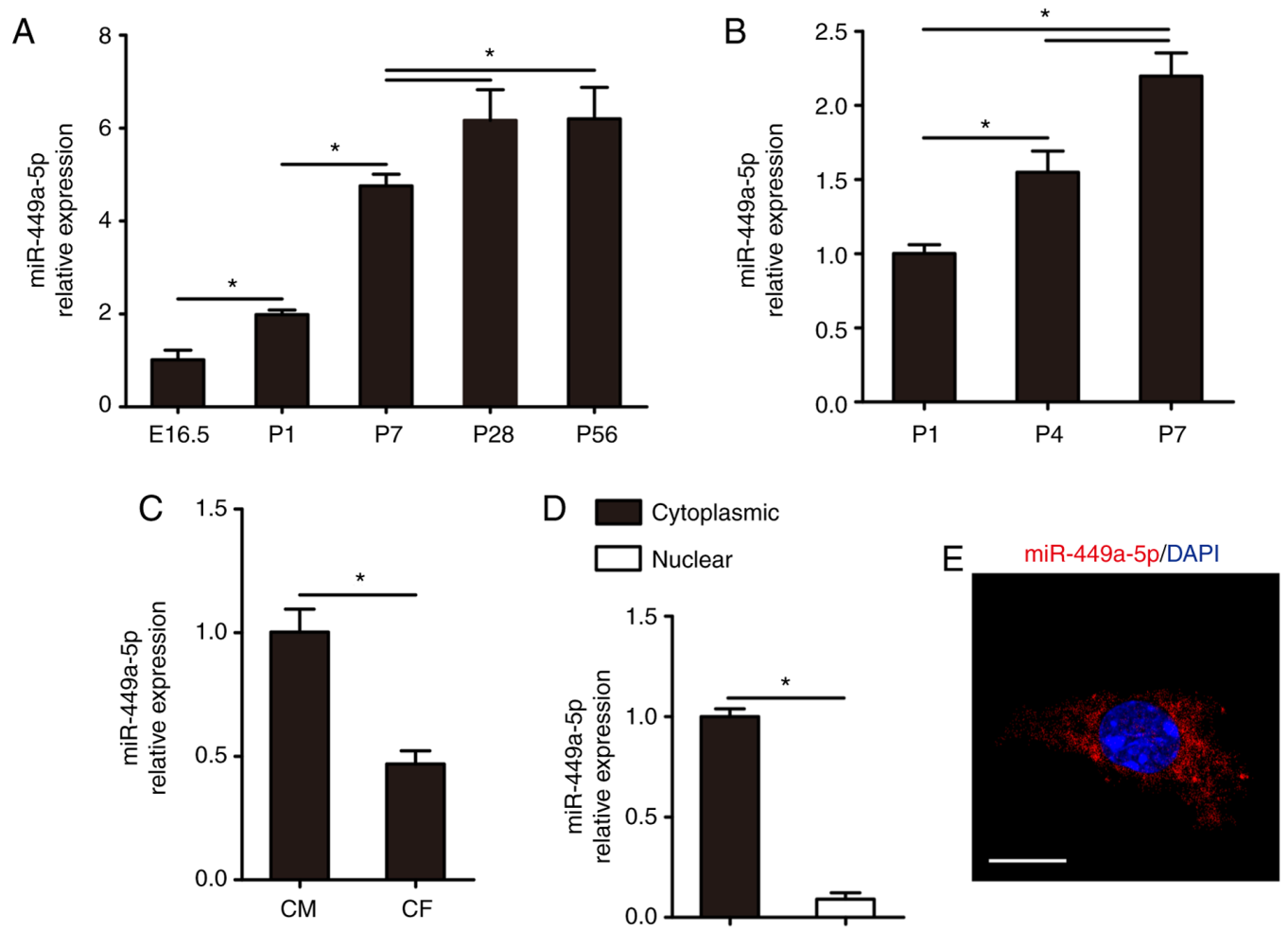

Figure 1. Expression of miR-449a-5p in mouse hearts and CMs. RT-qPCR results for miR-499a-5p expression in the (A) hearts from E16.5, P1, P7, P28 and P56 mice ( $\mathrm{n}=5$ ) and in (B) CMs isolated from P1, P4 and P7 mice ( $\mathrm{n}=3)$. (C) RT-qPCR results of miR-449a-5p expression in isolated P1 CMs and CFs ( $\mathrm{n}=3$ ). (D) Cytoplasmic and nuclear fractions for the abundance of miR-449a-5p expression in isolated P1 CMs (n=3). (E) RNA FISH assay results for miR-449a-5p detection in isolated P1 CMs (n=3). Scale bar, $20 \mu \mathrm{m}$. Statistical significance was calculated using one-way ANOVA followed by Tukey's test in (A), Least Significant Difference post hoc test in (B) and two-tailed unpaired Student's t-test in (C and D). Error bars represent mean \pm SEM. ${ }^{*} \mathrm{P}<0.05$ vs. indicated groups. miR, microRNA; CMs, cardiomyocytes; RT-qPCR, reverse transcription-quantitative PCR; E, embryonic; P, postnatal; CFs, cardiac fibroblasts.
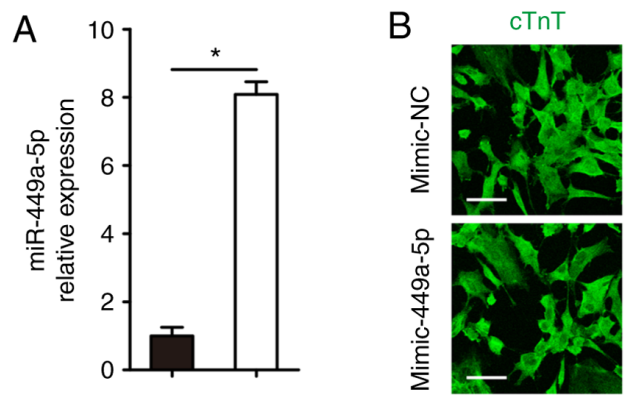

ki67

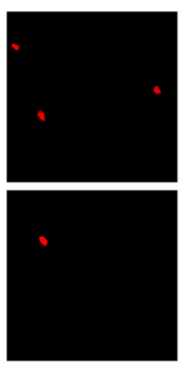

Hoechst
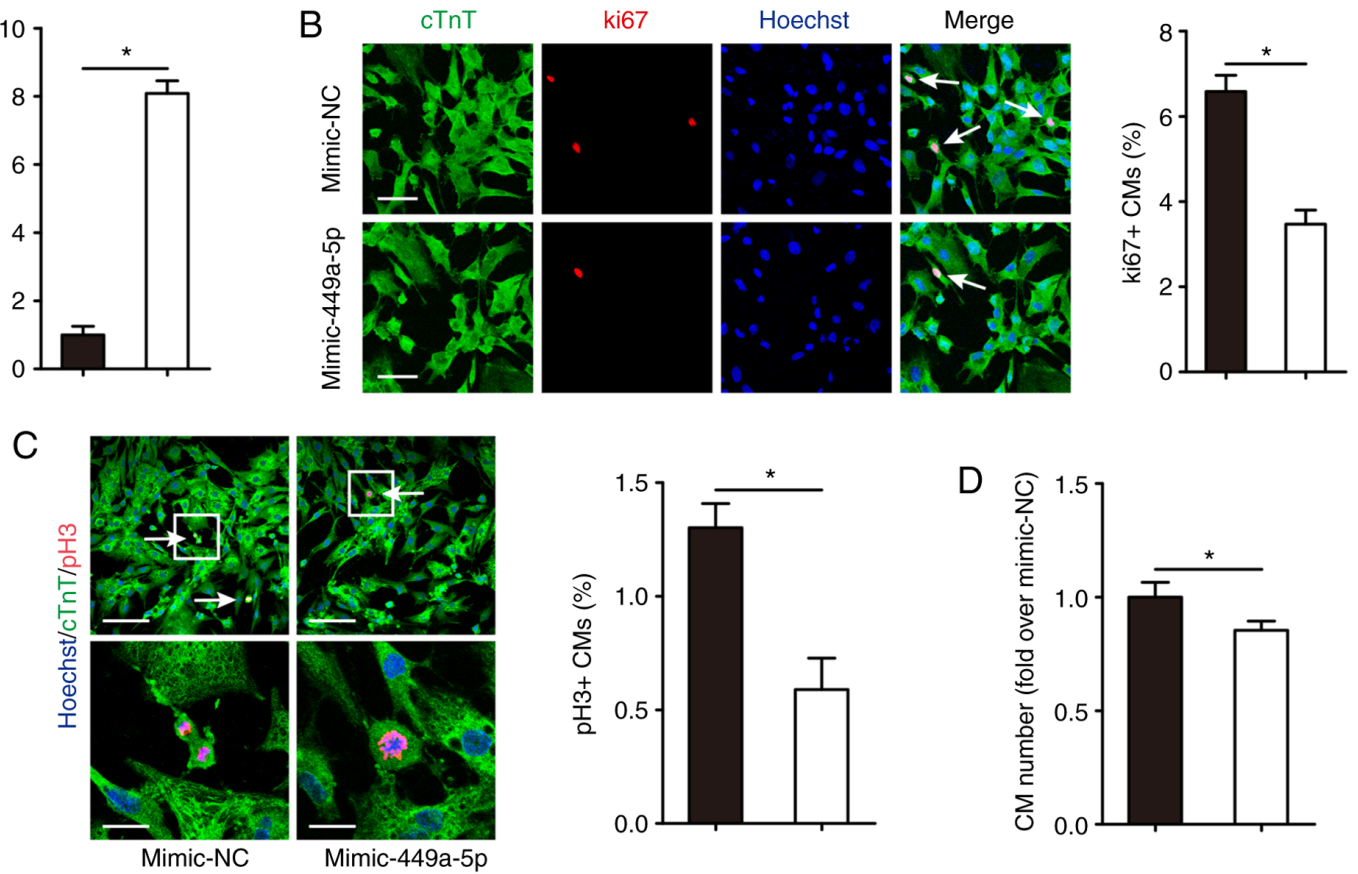

D

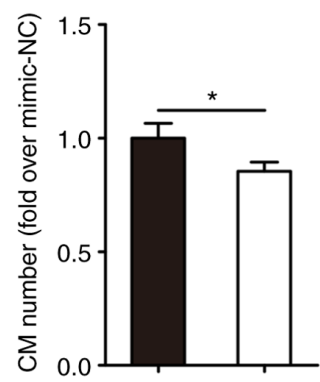

Figure 2. miR-449a-5p inhibits neonatal CM proliferation. Isolated P1 CMs were transfected with mimic-NCs or mimic-miR-499a-5p. (A) Reverse transcription-quantitative PCR results for the detection of miR-449a-5p expression $(n=3)$. (B) Ki67 immunofluorescence staining and quantification of ki67-positive CMs (n=3). Ki67-positive CMs were indicated by arrows. Scale bar, $50 \mu \mathrm{m}$. (C) pH3 immunofluorescence staining and quantification of pH3-positive CMs (n=3). pH3-positive CMs were indicated by arrows. Scale bar, $100 \mu \mathrm{m}$ (upper images) or $20 \mu \mathrm{m}$ (lower image). (D) Quantification of the number of CMs $(\mathrm{n}=3)$. Statistical significance was calculated using two-tailed unpaired Student's $\mathrm{t}$-test. Error bars represent mean \pm SEM. "P<0.05 vs. indicated groups. miR, microRNA; CM, cardiomyocyte; NC, negative control; cTnT, cardiac troponin T; pH3, histone H3 phosphorylated at serine 10. 

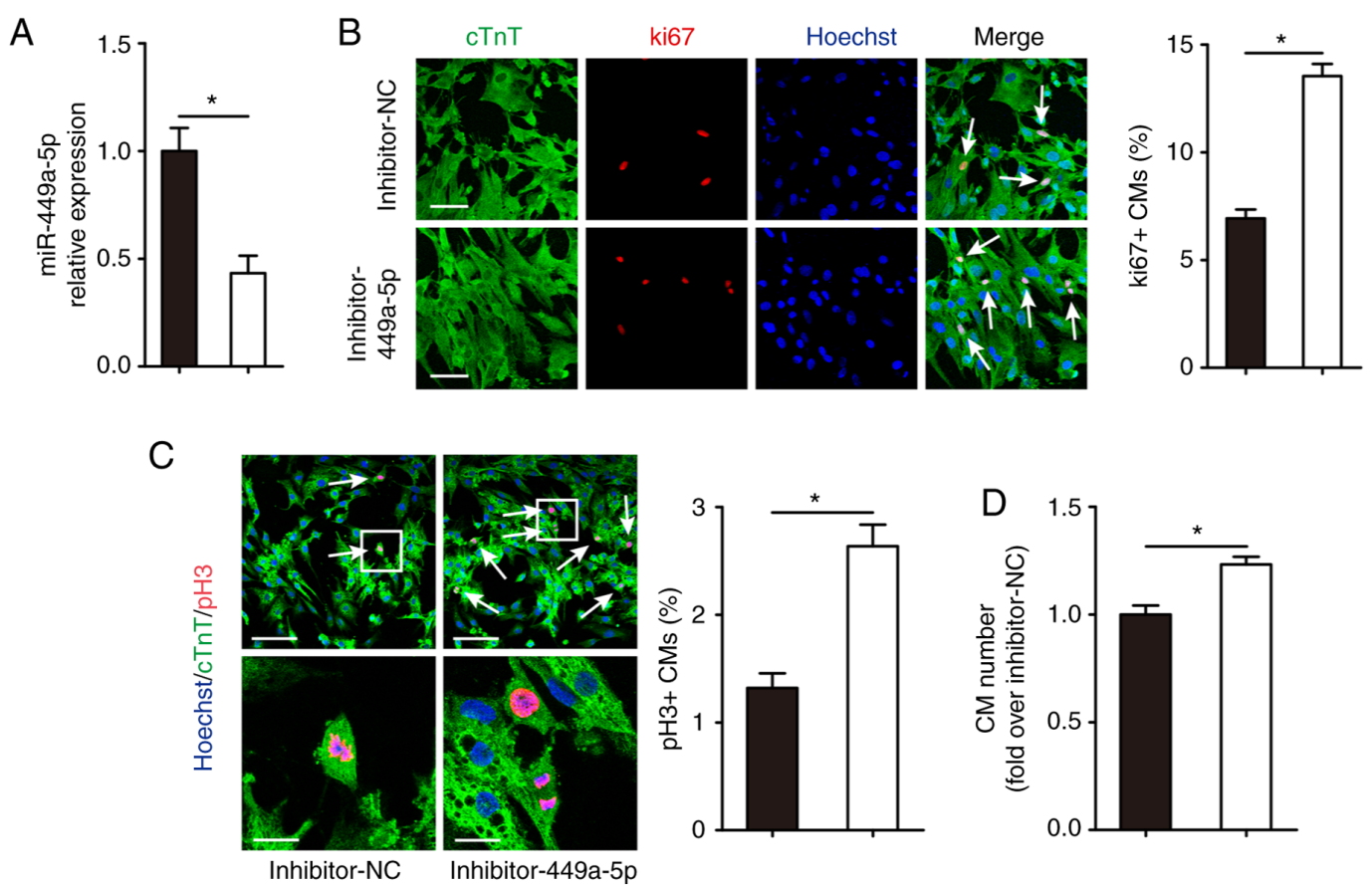

Figure 3. Knockdown of miR-449a-5p induces neonatal CM proliferation. Isolated P1 CMs were transfected with inhibitor-NCs or inhibitor-miR-449a-5p. (A) Reverse transcription-quantitative PCR results for detecting miR-449a-5p expression ( $\mathrm{n}=3$ ). (B) Ki67 immunofluorescence staining and quantification of ki67-positive CMs (n=3). Ki67-positive CMs were indicated by arrows. Scale bar, $50 \mu \mathrm{m}$. (C) pH3 immunofluorescence staining and quantification of pH3-positive CMs (n=3). Scale bar, $100 \mu \mathrm{m}$ (upper image) or $20 \mu \mathrm{m}$ (lower image). (D) Quantification of the numbers of CMs (n=3). Statistical significance was calculated using two-tailed unpaired Student's t-test. Error bars represent mean $\pm \mathrm{SEM}$. ${ }^{*} \mathrm{P}<0.05$ vs. indicated groups. miR, microRNA; CM, cardiomyocyte; NCs, negative controls; cTnT, cardiac troponin T; pH3, histone H3 phosphorylated at serine 10.

the luciferase activity of the reporter gene of CDK6 from $100.00 \pm 12.22$ to $55.63 \pm 6.77 \%$, whereas this reduction was abrogated when the seed sequences of the 3'-UTR binding site were mutated (Fig. 4B). These results suggested that CDK6 was a target for miR-449a-5p.

Subsequently, the endogenous efficiency of the RT-qPCR samples was tested according to a previous study that analyzed relative gene expression data using the $2^{-\Delta \Delta \mathrm{Cq}}$ method (27). The results revealed that the efficiency of primer amplification of CDK6 and $\beta$-actin was equal in the RT-qPCR experiments (Fig. S2). Therefore, $\beta$-actin as was used as the internal reference.

Then, whether miR-449a-5p had an effect on CDK6 in CMs was evaluated. CDK6 mRNA and protein expression was significantly decreased in isolated CMs transfected with mimic-miR-449a-5p compared with the NC group (Fig. 4C and D); however, the expression levels were significantly increased in the inhibitor-miR-449a-5p group compared with the inhibitor-NC group (Fig. 4C and D). These results indicated that miR-449a-5p bound directly to CDK6 mRNA and inhibited CDK6 expression.

CDK6 overexpression induces $C M$ proliferation. The effects of CDK6 on CM proliferation were evaluated. RT-qPCR confirmed that CDK6 mRNA expression significantly decreased during mouse heart development (Fig. 5A and B). Following this, the correlation between miR-449a-5p and CDK6 was determined by examining their expression levels in 15 ventricular heart samples from mice of different ages (E16.5, P1, P7, P28 or P56). A very strong negative correlation was found between miR-449a-5p and CDK6 mRNA expression levels $(r=-0.957)$ in the samples (Fig. 5C). Furthermore, it was detected whether CDK6 induced CM proliferation. RT-qPCR results demonstrated that CDK6 mRNA expression was upregulated in isolated CMs transfected with pcDNA-CDK6 compared with pcDNA-NC (Fig. 5D). Western blotting confirmed that CDK6 protein expression was upregulated in the pcDNA-CDK6 group compared with the pcDNA-NC group (Fig. 5E). CM proliferation was assessed via ki67 immunofluorescence staining, which revealed that CDK6 promoted CM proliferation in the pcDNA-CDK6-NC vs. the pcDNA-CDK6 group $(6.35 \pm 0.51 \%$ vs. $12.52 \pm 0.60 \%$, respectively; Fig. 5F).

miR-449a-5p inhibits CM proliferation by targeting CDK6. Whether CDK6 was involved in the functional role of miR-449a-5p in CM proliferation was also investigated. Mimic-miR-449a-5p and pcDNA-CDK6 were co-transfected into isolated P1 CMs. RT-qPCR and western blotting results demonstrated that CDK6 mRNA and protein expression was significantly decreased in CMs transfected with mimic-miR-449a-5p alone compared with the control group, but significantly increased in the mimic-miR-449a-5p + pcDNA-CDK6 group compared with the mimic-miR-449a-5p group (Fig. 6A and B). The ki67 immunofluorescence staining assay identified 


\begin{tabular}{|c|c|c|}
\hline mRNA & Matching & Position \\
\hline $\begin{array}{l}\text { CDK6 } \\
\text { 3'UTR } \\
\text { site } 1\end{array}$ & 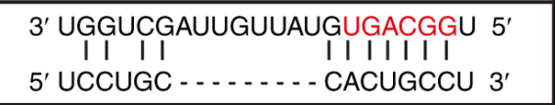 & $1079-1092$ \\
\hline $\begin{array}{l}\text { CDK6 } \\
\text { 3'UTR } \\
\text { site } 2\end{array}$ & $\begin{array}{l}\text { 3' UGGUCGAU - UGUUA - - UGUGACGGU 5' } \\
\text { I I I I I I : I I I I I I I I I I I } \\
\text { 5' CACAGCUCUGCUGCCGACACUGCCA 3' }\end{array}$ & 2185-2209 \\
\hline
\end{tabular}
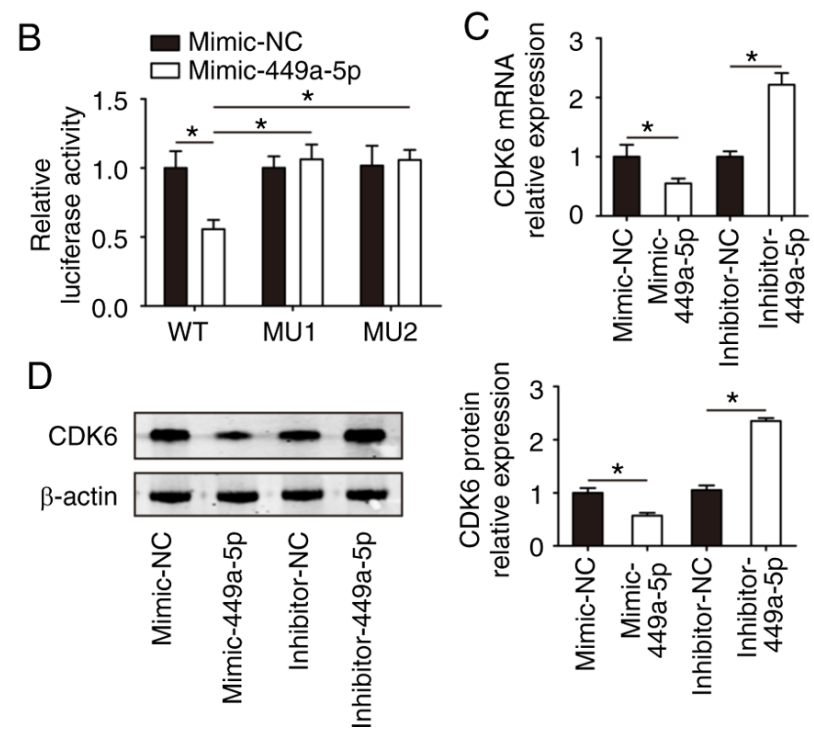

Figure 4.CDK6 is a target gene of miR-449a-5p. (A) Binding sites for miR-449a-5p on the 3'-UTR of CDK6 as predicted using miRmap and miRanda. (B) Luciferase assays of isolated P1 CMs transfected with luciferase-CDK6-WT, luciferase-CDK6-MU site 1 and luciferase-CDK6-MU site 2 (n=3). (C) Reverse transcription-quantitative PCR assays detected CDK6 mRNA expression in isolated P1 CMs transfected with mimic-NC, mimic-miR-449a-5p, inhibitor-NC or inhibitor-miR-449a-5p $(\mathrm{n}=3)$. (D) Western blotting results and semi-quantitative analyses of CDK6 protein expression in isolated P1 CMs transfected with mimic-NC, mimic-miR-449a-5p, inhibitor-NC or inhibitor-miR-449a-5p ( $\mathrm{n}=3$ ). Statistical significance was calculated using one-way ANOVA followed by Tukey's test in (B) or Least Significant Difference post hoc test in (C and D) Error bars represent mean \pm SEM. "P<0.05 vs. indicated groups. miR, microRNA; UTR, untranslated region; P, postnatal; CMs, cardiomyocytes; WT, wild-type; MU, mutant; NC, negative control.
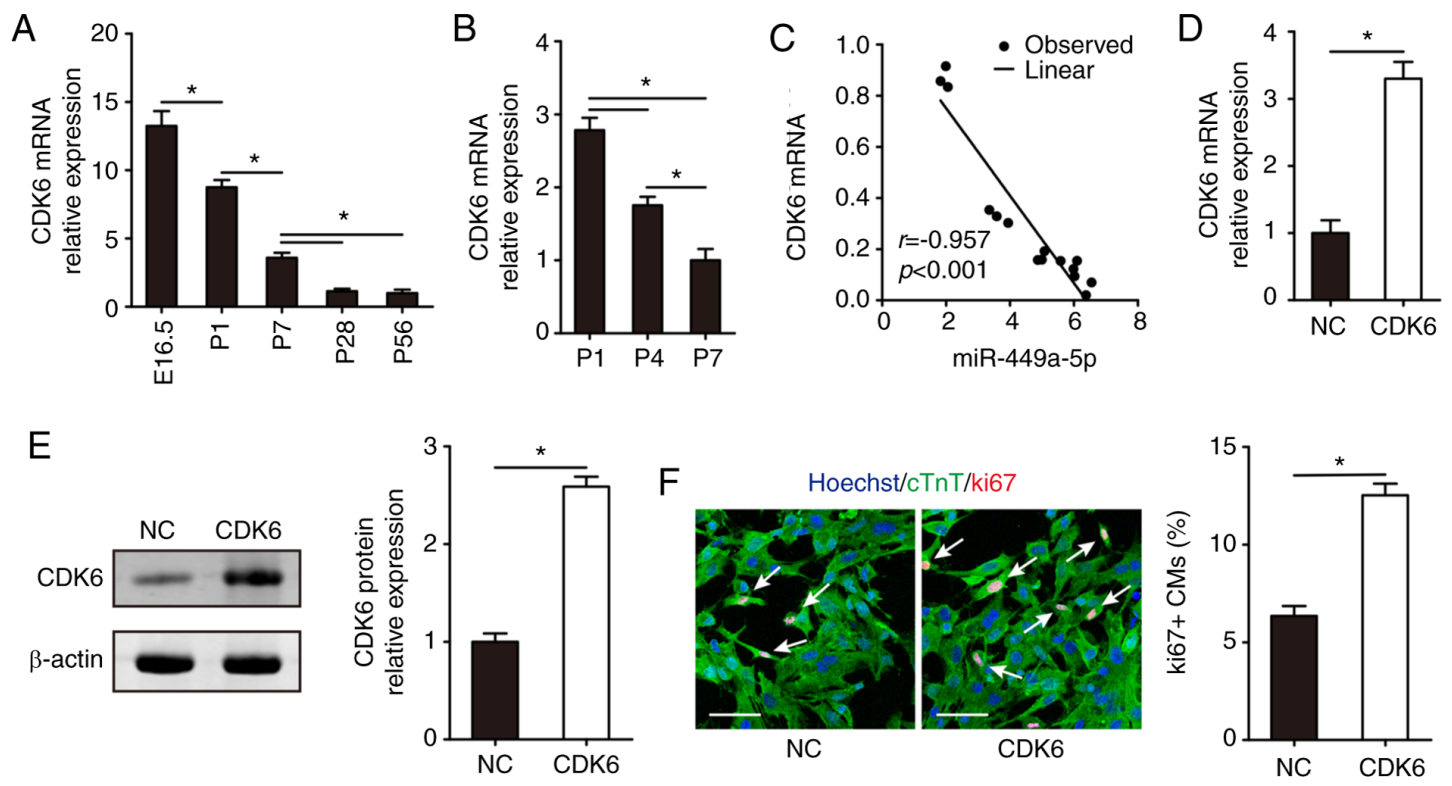

Figure 5. CDK6 overexpression promotes CM proliferation. (A) Reverse transcription-quantitative PCR results of (A) CDK6 mRNA expression in the hearts of E16.5, P1, P7, P28 and P56 mice (n=5) and (B) CDK6 mRNA expression in CMs isolated from P1, P4 and P7 mice (n=3). (C) Correlation between miR-449a-5p and CDK6 mRNA expression levels in 15 ventricular heart samples from different aged mice. (D) CDK6 mRNA expression in isolated P1 CMs transfected with $\mathrm{NC}$ or pcDNA-CDK6 $(\mathrm{n}=3)$. (E) Western blotting results and semi-quantitative analyses of CDK6 protein expression in isolated P1 CMs transfected with NC or pcDNA-CDK6 (n=3). (F) Ki67 immunofluorescence staining in isolated P1 CMs transfected with NC or pcDNA-CDK6 and quantification of ki67-positive CMs (n=3). Ki67-positive CMs were indicated by arrows. Scale bar, $50 \mu \mathrm{m}$. Statistical significance was calculated using one-way ANOVA followed by Tukey's test in (A), Least Significant Difference post hoc test in (B), Spearman's test in (C) and two-tailed unpaired Student's t-test in (D-F). Error bars represent mean \pm SEM. "P<0.05 vs. indicated groups. CM, cardiomyocyte; E, embryonic; P, postnatal; miR, microRNA; NC, negative control; cTnT, cardiac troponin T. 
A

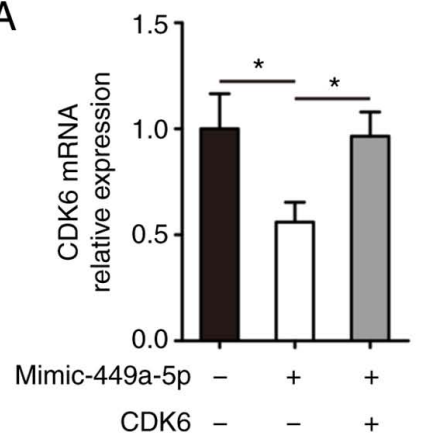

C
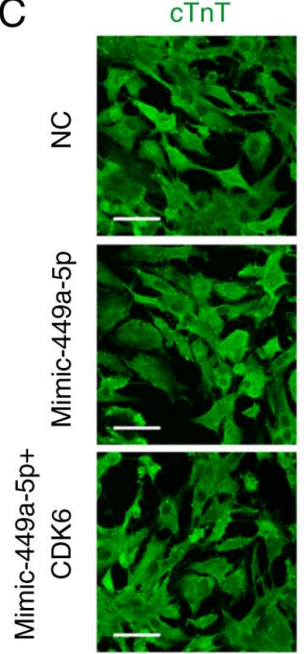

ki67
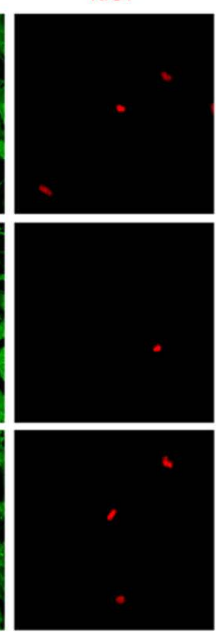

B

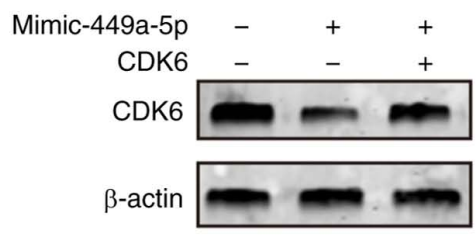

Hoechst
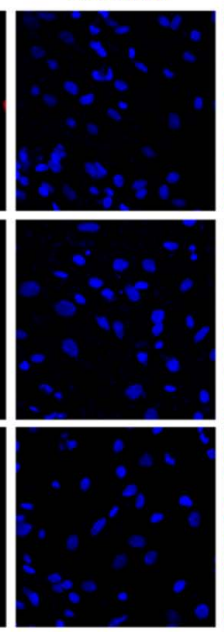

Merge

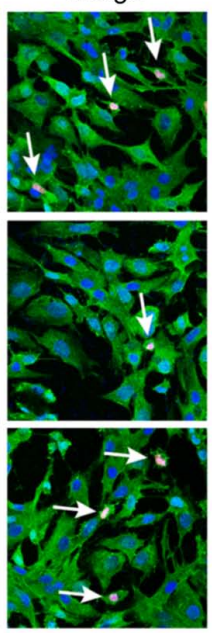

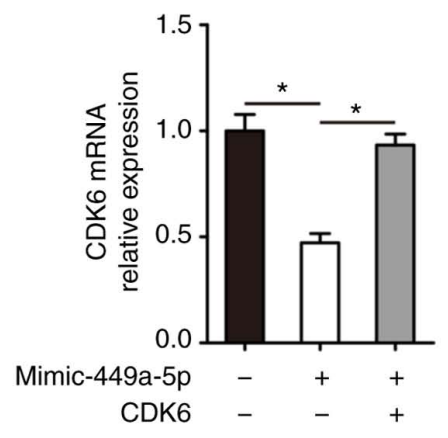

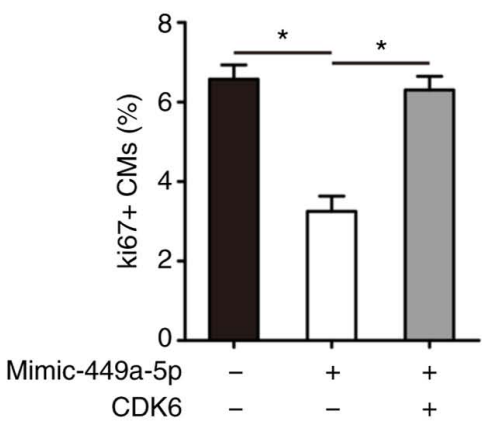

Figure 6. CDK6 is involved in the inhibitory effects of miR-449a-5p on CM proliferation. P1 CMs were transfected with NC, mimic-miR-449a-5p or mimic-miR-449a-5p + pcDNA-CDK6 ( $\mathrm{n}=3$ /group). (A) Reverse transcription-quantitative PCR results for the detection of CDK6 mRNA expression. (B) Western blotting results and semi-quantitative analyses of CDK6 protein expression. (C) Ki67 immunofluorescence staining and quantification of ki67-positive CMs. Ki67-positive CMs were indicated by arrows. Scale bar, $50 \mu \mathrm{m}$. Statistical significance was calculated using one-way ANOVA followed by Least Significant Difference post hoc test in A-C. Error bars represent mean \pm SEM. "P $<0.05$ vs. indicated groups. miR, microRNA; CM, cardiomyocyte; $\mathrm{P}$, postnatal; NC, negative control; cTnT, cardiac troponin T.

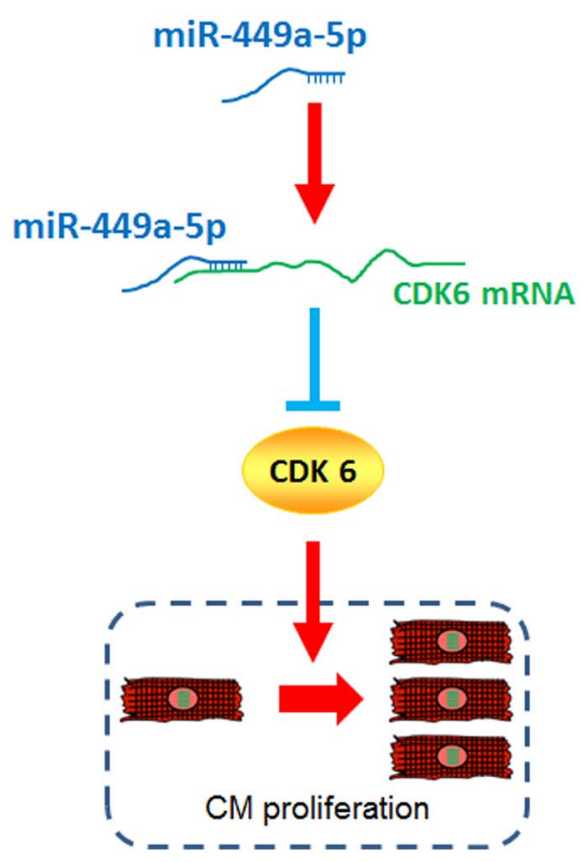

Figure 7. Schematic diagram of miR-449a-5p inhibiting CM proliferation. miR-449a-5p directly binds to CDK6 and reduces the expression of CDK6 proteins, resulting in the repression of CM proliferation. miR, microRNA; $\mathrm{CM}$, cardiomyocyte. that co-transfection with the mimic-miR-449a-5p and pcDNA-CDK6 abrogated the decline in ki67-positive CMs induced by mimic-miR-449a-5p $(6.30 \pm 0.34 \%$ vs. $3.25 \pm 0.38 \%$, respectively; Fig. $6 \mathrm{C})$. Collectively, the results indicated that CDK6 mediated the regulatory role of miR-449a-5p in CM proliferation.

\section{Discussion}

The present study demonstrated that miR-449a-5p was a negative regulator of CM proliferation. Additionally, miR-449a-5p directly bound the CDK6 mRNA 3'-UTR and inhibited CDK6 expression. Thus, it was suggested that CDK6 mediated miR-449a-5p-inhibited CM proliferation (Fig. 7). These findings indicated that miR-449a-5p may be a valuable therapeutic target for activating cardiac regeneration in the treatment cardiac diseases.

Increasing numbers of miRNAs, including miR-17-92 (12), miR-128 (36) and miR-590 (11), have been observed to regulate $\mathrm{CM}$ proliferation. miR-449a-5p has been reported to be associated with CM hypoxia/reoxygenation injury (18). miR-449a-5p and miR-34a contain distinct seed regions, which suggests that miR-449a-5p and miR-34a may have similar functions $(19,37)$. miR-34a has been proven to regulate 
$\mathrm{CM}$ proliferation and cardiac regeneration in post-myocardial infarction (10). Therefore, it was hypothesized that miR-449a-5p may regulate CM proliferation. miR-449a-5p has been identified to negatively regulate $\mathrm{CM}$ proliferation from previous high-throughput functional screening data (11). In the present study, by quantifying miR-449a-5p expression levels in CMs in mice at different ages, miR-449a-5p was found to be increased during CM proliferation, accompanied by the gradual decline in the proliferative ability of CMs. There was low miR-449a-5p expression in the embryonic heart, increased expression during the neonatal period and very high expression at the adult stages.

To further investigate whether miR-449a-5p regulated $\mathrm{CM}$ proliferation, $\mathrm{CM}$ proliferation in isolated mouse $\mathrm{CMs}$ was evaluated using labeling proliferation-related markers. CMs were immunostained with previously used markers, ki67 and $\mathrm{pH} 3$, which are conventional markers of CM proliferation and mitosis, respectively $(11,31)$. The present results suggested that miR-449a-5p overexpression led to a significant decrease in the percentage of ki67- and $\mathrm{pH} 3$-positive $\mathrm{CMs}$, as well as the numbers of CMs. However, knockdown of miR-449a-5p caused an increase in ki67- and pH3-positive CMs. The present study also investigated whether miR-449a-5p caused physiological myocardial growth and development or myocardial damage. Changes in stem/progenitor markers RUNX1, c-kit and DAB2 expression levels and in CM apoptosis were detected using TUNEL staining. The results demonstrated that miR-449a-5p overexpression inhibited CM dedifferentiation markers and induced CM apoptosis. Consistent with the present results, previous studies have reported that miR-449a-5p inhibition protected CMs against apoptosis $(18,38)$. These results support the current hypothesis that miR-449a-5p inhibits CM proliferation.

The present study found that miR-449a-5p regulated CM proliferation by targeting CDK6. CDK6 is a cyclin D activated kinase that phosphorylates the $R b$ in the $G_{1}$ phase and associates with E2F to regulate $G_{1}$ to $S$ transition in the cell cycle (21). Consistent with previous studies $(39,40)$, the current results demonstrated that the expression of CDK6 was high in the embryonic heart, decreased during the neonatal period and was very low at the adult stages. Thus, the expression pattern of CDK6 was opposite to that of miR-449a-5p. A previous study reported that miR-449a-5p directly targets CDK6 to suppress cell proliferation in neuroblastoma (16). The current study also observed that CDK6 was a target gene of miR-449a-5p in CM proliferation. Additionally, the present results identified that CDK6 overexpression promoted $\mathrm{CM}$ proliferation, and the overexpression of CDK6 rescued the inhibitory effects caused by miR-449a-5p in CM proliferation. Collectively, these results suggested that miR-449a-5p inhibited CM proliferation via decreased CDK6 expression.

The loss of CMs and their deficient regeneration capability are major contributors to the pathogenesis of numerous cardiac diseases, such as heart failure $(41,42)$. Promoting CM proliferation is hypothesized to be one of the most effective regenerative therapeutic strategies to prevent exacerbation of heart failure $(3,4)$. Previous studies have revealed that the inhibition of negative miRNA regulators of proliferation, including the miR-15 family (43) or, conversely, the enhancement of proliferative miRNAs, such as miR-199a and -590 (11), lead to increased $\mathrm{CM}$ proliferation and improved cardiac function post-myocardial injury. Combined with the current results, these research studies highlight the mechanistic and therapeutic significance of miRNA regulation in CM proliferation. Therefore, treating the injured heart directly with miRNAs with regenerative abilities or targeting miRNA with small molecular drugs may improve cardiac function in patients with cardiac injury $(44,45)$.

The present study had several limitations. Firstly, although the effects of miR-449a-5p on CM proliferation were verified in vitro, additional comprehensive experiments in vivo may aid to further detect the role of miR-449a-5p. Secondly, whether miR-449a-5p regulates cardiac regeneration in myocardial infarction and heart failure should be clarified in future studies.

In conclusion, the present study identified that miR-449a-5p regulated CM proliferation by inhibiting CDK6. These results indicated that miR-449a-5p may act as a novel effective gene target for treatment approaches for myocardial injury.

\section{Acknowledgements}

Not applicable.

\section{Funding}

The current work was supported by grants from the Science and Technology Plan of Guizhou Province [Guizhou Science and Technology Cooperation Foundation; grant no. (2019)1260] and the National Natural Science Foundation of China (grant no. 81960083).

\section{Availability of data and materials}

The datasets used and/or analyzed during the current study are available from the corresponding author on reasonable request.

\section{Authors' contributions}

BL and XS conceived the current study and designed experiments. BL, FY, JH and XH performed the experiments. ZW, SD and MT analyzed the data. BL and XS wrote the manuscript. All authors read and approved the final manuscript.

\section{Ethics approval and consent to participate}

The animal study was approved by the Guizhou University Subcommittee of Experimental Animal Ethics.

\section{Patient consent for publication}

Not applicable.

\section{Competing interests}

The authors declare that they have no competing interests.

\section{References}

1. Organization WH: The top 10 causes of death. https://www.who. $\mathrm{int} / \mathrm{en} /$ news-room/fact-sheets/detail/the-top-10-causes-of-death, 2018.

2. Li M and Izpisua Belmonte JC: Mending a faltering heart. Circ Res 118: 344-351, 2016. 
3. Yuan X and Braun T: Multimodal regulation of cardiac myocyte proliferation. Circ Res 121: 293-309, 2017.

4. Leach JP and Martin JF: Cardiomyocyte proliferation for therapeutic regeneration. Curr Cardiol Rep 20: 63, 2018.

5. Li B, Hu Y, Li X, Jin G, Chen X, Chen G, Chen Y, Huang S, Liao W, Liao Y, et al: Sirtl antisense long noncoding RNA promotes cardiomyocyte proliferation by enhancing the stability of Sirt1. J Am Heart Assoc 7: e009700, 2018.

6. Wojciechowska A, Braniewska A and Kozar-Kaminska K MicroRNA in cardiovascular biology and disease. Adv Clin Exp Med 26: 865-874, 2017.

7. Bernardo BC, Ooi JY, Lin RC and McMullen JR: miRNA therapeutics: A new class of drugs with potential therapeutic applications in the heart. Future Med Chem 7: 1771-1792, 2015.

8. Colpaert RMW and Calore M: MicroRNAs in cardiac diseases. Cells 8: 737, 2019.

9. Tian Y, Liu Y, Wang T, Zhou N, Kong J, Chen L, Snitow M, Morley M, Li D, Petrenko N, et al: A microRNA-Hippo pathway that promotes cardiomyocyte proliferation and cardiac regeneration in mice. Sci Transl Med 7: 279ra238, 2015.

10. Yang Y, Cheng HW, Qiu Y, Dupee D, Noonan M, Lin YD, Fisch S, Unno K, Sereti KI and Liao R: MicroRNA-34a plays a key role in cardiac repair and regeneration following myocardial infarction. Circ Res 117: 450-459, 2015.

11. Eulalio A, Mano M, Dal Ferro M, Zentilin L, Sinagra G, Zacchigna S and Giacca M: Functional screening identifies miRNAs inducing cardiac regeneration. Nature 492: 376-381, 2012.

12. Chen J, Huang ZP, Seok HY, Ding J, Kataoka M, Zhang Z, Hu X, Wang G, Lin Z, Wang S, et al: mir-17-92 cluster is required for and sufficient to induce cardiomyocyte proliferation in postnatal and adult hearts. Circ Res 112: 1557-1566, 2013.

13. Liang D, Li J, Wu Y, Zhen L, Li C, Qi M, Wang L, Deng F, Huang J, Lv F, et al: miRNA-204 drives cardiomyocyte proliferation via targeting Jarid2. Int J Cardiol 201: 38-48, 2015.

14. Bou Kheir T, Futoma-Kazmierczak E, Jacobsen A, Krogh A Bardram L, Hother C, Grønbaek K, Federspiel B, Lund AH and Friis-Hansen L: miR-449 inhibits cell proliferation and is down-regulated in gastric cancer. Mol Cancer 10: 29, 2011

15. Sandbothe M, Buurman R, Reich N, Greiwe L, Vajen B, Gürlevik E, Schäffer V, Eilers M, Kühnel F, Vaquero A, et al: The microRNA-449 family inhibits TGF- $\beta$-mediated liver cancer cell migration by targeting SOX4. J Hepatol 66: 1012-1021, 2017.

16. Zhao Z, Ma X, Sung D, Li M, Kosti A, Lin G, Chen Y, Pertsemlidis A, Hsiao TH and Du L: microRNA-449a functions as a tumor suppressor in neuroblastoma through inducing cell differentiation and cell cycle arrest. RNA Biol 12: 538-554, 2015

17. Yong-Ming H, Ai-Jun J, Xiao-Yue X, Jian-Wei L, Chen Y and Ye C: miR-449a: A potential therapeutic agent for cancer Anticancer Drugs 28: 1067-1078, 2017.

18. Cheng J, Wu Q, Lv R, Huang L, Xu B, Wang X, Chen A and He F: MicroRNA-449a inhibition protects H9C2 cells against Hypoxia/Reoxygenation-induced injury by targeting the Notch-1 signaling pathway. Cell Physiol Biochem 46: 2587-2600, 2018.

19. Song R, Walentek P, Sponer N, Klimke A, Lee JS, Dixon G, Harland R, Wan Y, Lishko P, Lize M, et al: miR-34/449 miRNAs are required for motile ciliogenesis by repressing cp110. Nature 510: 115-120, 2014

20. Chen H, Lin YW, Mao YQ, Wu J, Liu YF, Zheng XY and Xie LP: MicroRNA-449a acts as a tumor suppressor in human bladder cancer through the regulation of pocket proteins. Cancer Lett 320: 40-47, 2012.

21. Ezhevsky SA, Nagahara H, Vocero-Akbani AM, Gius DR, Wei MC and Dowdy SF: Hypo-phosphorylation of the retinoblastoma protein $(\mathrm{pRb})$ by cyclin D:Cdk4/6 complexes results in active pRb. Proc Nati Acad Sci USA 94: 10699-10704, 1997.

22. National Research Council Committee for the Update of the Guide for the $\mathrm{C}$ and Use of Laboratory A: The National Academies Collection: Reports funded by National Institutes of Health. In: Guide for the Care and Use of Laboratory Animals National Academies Press (US), Copyright (C) 2011, National Academy of Sciences, Washington (DC), 2011. https://www.ncbi nlm.nih.gov/books/NBK4119/. Accessed Feb 02, 2020.

23. Ehler E, Moore-Morris T and Lange S: Isolation and culture of neonatal mouse cardiomyocytes. J Vis Exp 79: 50154, 2013.

24. Mbogo GW, Nedeva C and Puthalakath H: Isolation of cardiomyocytes and cardiofibroblasts for ex vivo analysis. Methods Mol Biol 1419: 117-129, 2016.
25. Li X, He X, Wang H, Li M, Huang S, Chen G, Jing Y, Wang S, Chen Y, Liao W, et al: Loss of AZIN2 splice variant facilitates endogenous cardiac regeneration. Cardiovasc Res 114: 1642-1655, 2018.

26. Liu X, Xiao J, Zhu H, Wei X, Platt C, Damilano F, Xiao C, Bezzerides V, Bostrom P, Che L, et al: miR-222 is necessary for exercise-induced cardiac growth and protects against pathological cardiac remodeling. Cell Metab 21: 584-595, 2015.

27. Livak KJ and Schmittgen TD: Analysis of relative gene expression data using real-time quantitative PCR and the 2(-Delta Delta C(T)) method. Methods 25: 402-408, 2001

28. Yang J, Gong Y, Cai J, Liu Q and Zhang Z: Lnc-3215 suppression leads to calcium overload in selenium deficiency-induced chicken heart lesion via the lnc-3215-miR-1594-TNN2 pathway. Mol Ther Nucleic Acids 18: 1-15, 2019.

29. Vejnar CE and Zdobnov EM: MiRmap: Comprehensive prediction of microRNA target repression strength. Nucleic Acids Res 40: 11673-11683, 2012

30. Enright AJ, John B, Gaul U, Tuschl T, Sander C and Marks DS: MicroRNA targets in Drosophila. Genome Biol 5: R1, 2003.

31. D'Uva G, Aharonov A, Lauriola M, Kain D, Yahalom-Ronen Y, Carvalho S, Weisinger K, Bassat E, Rajchman D, Yifa O, et al: ERBB2 triggers mammalian heart regeneration by promoting cardiomyocyte dedifferentiation and proliferation. Nat Cell Biol 17: 627-638, 2015.

32. Kubin T, Pöling J, Kostin S, Gajawada P, Hein S, Rees W, Wietelmann A, Tanaka M, Lörchner H, Schimanski S, et al: Oncostatin $\mathrm{M}$ is a major mediator of cardiomyocyte dedifferentiation and remodeling. Cell Stem Cell 9: 420-432, 2011

33. Bartel DP: MicroRNAs: Genomics, biogenesis, mechanism, and function. Cell 116: 281-297, 2004.

34. Sherr CJ, Beach D and Shapiro GI: Targeting CDK4 and CDK6: From discovery to therapy. Cancer Discov 6: 353-367, 2016.

35. Tigan AS, Bellutti F, Kollmann K, Tebb G and Sexl V: CDK6-a review of the past and a glimpse into the future: From cell-cycle control to transcriptional regulation. Oncogene 35: 3083-3091, 2016.

36. Huang W, Feng Y, Liang J, Yu H, Wang C, Wang B, Wang M, Jiang L, Meng W, Cai W, et al: Loss of microRNA-128 promotes cardiomyocyte proliferation and heart regeneration. Nat Commun 9: 700, 2018.

37. Mercey O, Popa A, Cavard A, Paquet A, Chevalier B, Pons N, Magnone V, Zangari J, Brest P, Zaragosi LE,s: Characterizing isomiR variants within the microRNA-34/449 family. FEBS Lett 591: 693-705, 2017

38. Zhang X, Dong H, Liu Y, Han J, Tang S and Si J: Tetramethylpyrazine partially relieves hypoxia-caused damage of cardiomyocytes H9c2 by downregulation of miR-449a. J Cell Physiol: Feb 15,2019 (Epub ahead of print). doi: 10.1002/jcp.28151.

39. Kim WH, Joo CU, Ku JH, Ryu CH, Koh KN, Koh GY and Ko JK: Cell cycle regulators during human atrial development. Korean J Intern Med 13: 77-82, 1998.

40. Ponnusamy M, Li PF and Wang K: Understanding cardiomyocyte proliferation: An insight into cell cycle activity. Cell Mol Life Sci 74: 1019-1034, 2017.

41. Gill C, Mestril R and Samali A: Losing heart: The role of apoptosis in heart disease-a novel therapeutic target? FASEB J 16: 135-146, 2002.

42. Petrovic D: Cytopathological basis of heart failure-cardiomyocyte apoptosis, interstitial fibrosis and inflammatory cell response. Folia Biol (Praha) 50: 58-62, 2004.

43. Porrello ER, Mahmoud AI, Simpson E, Johnson BA, Grinsfelder D, Canseco D, Mammen PP, Rothermel BA, Olson EN and Sadek HA: Regulation of neonatal and adult mammalian heart regeneration by the miR-15 family. Proc Natl Acad Sci USA 110: 187-192, 2013.

44. Lock MC and Tellam RL: The role of miRNA regulation in fetal cardiomyocytes, cardiac maturation and the risk of heart disease in adults. The J Physiol 596: 5625-5640, 2018.

45. Giacca $M$ and Zacchigna S: Harnessing the microRNA pathway for cardiac regeneration. J Mol Cell Cardiol 89: 68-74, 2015.

This work is licensed under a Creative Commons Attribution-NonCommercial-NoDerivatives 4.0 International (CC BY-NC-ND 4.0) License. 